\title{
The nematic-disordered phase transition in systems of long rigid rods on two dimensional lattices
}

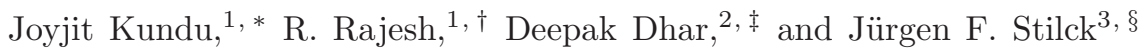 \\ ${ }^{1}$ The Institute of Mathematical Sciences, C.I.T. Campus, Taramani, Chennai 600113, India \\ ${ }^{2}$ Department of Theoretical Physics, Tata Institute of Fundamental Research, Homi Bhabha Road, Mumbai 400005, India \\ ${ }^{3}$ Instituto de Física and National Institute of Science and Technology for Complex Systems, \\ Universidade Federal Fluminense, Av. Litorânea s/n, 24210-346 Niterói, RJ, Brazil
}

(Dated: October 10, 2018)

\begin{abstract}
We study the phase transition from a nematic phase to a high-density disordered phase in systems of long rigid rods of length $k$ on the square and triangular lattices. We use an efficient Monte Carlo scheme that partly overcomes the problem of very large relaxation times of nearly jammed configurations. The existence of a continuous transition is observed on both lattices for $k=7$. We study correlations in the high-density disordered phase, and we find evidence of a crossover length scale $\xi^{*} \gtrsim 1400$, on the square lattice. For distances smaller than $\xi^{*}$, correlations appear to decay algebraically. Our best estimates of the critical exponents differ from those of the Ising model, but we cannot rule out a crossover to Ising universality class at length scales $\gg \xi^{*}$. On the triangular lattice, the critical exponents are consistent with those of the two dimensional three-state Potts universality class.

PACS numbers: 64.60.Cn,64.70.mf,05.50.+q
\end{abstract}

\section{INTRODUCTION}

The study of the ordering transition in a system of long rigid rods in solution with only excluded volume interaction has a long history, starting with Onsager's proof that beyond a critical density, a solution of thin cylindrical rods would undergo a transition to an orientationally ordered state [1 $[4]$. In two dimensional continuum space, when the rods may orient in any direction, the continuous rotational symmetry remains unbroken at any density. However, the system undergoes a KosterlitzThouless type transition from a low-density phase with exponential decay of orientational correlations to a highdensity phase with a power law decay [5 8 ].

In this paper, we study the problem when the underlying space is discrete: the square or the triangular lattice. Straight rods occupying $k$ consecutive sites along any one lattice direction will be called $k$-mers. For dimers $(k=2)$, it has been shown rigorously that the system remains in the isotropic phase at all packing densities [9]. A system of dimers with additional interactions, either attractive, favoring parallel alignment, or repulsive, disallowing nearest neighbor occupation, can have ordered phases [10]. For $k>2$, the existence of a phase transition, with only hard-core interactions, remained unsettled for a long time [11]. Ghosh and Dhar recently argued that $k$-mers on a square lattice, for $k \geq k_{\text {min }}$, would undergo two phase transitions, and the nematic phase would exist for only an intermediate range of densities $\rho_{1}^{*}<\rho<\rho_{2}^{*}$

\footnotetext{
*Electronic address: joyjit@imsc.res.in

${ }^{\dagger}$ Electronic address: rrajesh@imsc.res.in

${ }^{\ddagger}$ Electronic address: ddhar@theory.tifr.res.in

$\S$ Electronic address: jstilck@if.uff.br
}

[12]. Similar behavior is expected in higher dimensions. In two dimensions, numerical studies have shown that $k_{\min }=7$ [12]. The existence of the nematic phase, and hence the first transition from the isotropic to nematic phase, has been proved rigorously 13]. This transition has also been studied in detail through Monte Carlo simulations [14 17]. On the square lattice, the transition is numerically found to be in the Ising (equivalently the liquid-gas) universality class [14, 18], and on the triangular lattices, it is in the $q=3$ Potts model universality class 14, 15].

In this paper, we investigate whether the high-density disordered phase is a reentrant low-density disordered phase, or a new qualitatively distinct phase. To distinguish between these two phases without nematic order, we will refer to the first as low-density disordered (LDD) phase and the second as high-density disordered (HDD) phase in the remainder of the paper.

The second transition at $\rho_{2}^{*}$ from the nematic to the HDD phase has not been studied much so far. Numerical studies are difficult because of the large relaxation times of the nearly jammed configurations at high densities. Conventional Monte Carlo algorithms using depositionevaporation moves involving only addition or removal of single rods at a time are quite inefficient at large densities. With additional diffusion and rotation moves, it is possible to equilibrate the system [17, 19], but the algorithm is still not efficient enough to make quantitative studies of the transition, or the nature of the HDD phase. In Ref. [12], a variational estimate of the entropy of the nematic and the HDD phases suggests that $1-\rho_{2}^{*}$ should vary as $1 / k^{2}$ for large $k$. Linares et. al. estimated that $0.87 \leq \rho_{2}^{*} \leq 0.93$ for $k=7$, and proposed an approximate functional form for the entropy as a function of the density [17]. However, not much is known about the nature of transition. Recently, we showed that a Bethe- 
like approximation becomes exact on a random locally tree like layered lattice, and for the 4-coordinated lattice, $k_{\min }=4$. But on this lattice, the second transition is absent [20].

By implementing an efficient Monte Carlo algorithm, we show that, for $k=7$, at high densities the orientational order is absent on both square and triangular lattices. We investigate the nature of this HDD phase. Using lattices of size up to $L=2576$, we find evidence of a power-law decay of orientational correlations between rods for distances $r \leq \xi^{*} \approx 1400$, where $\xi^{*}$ is a characteristic length scale of the system. Correlations appear to decay faster for distances $r \gtrsim \xi^{*}$, but we have limited data in this regime, and cannot rule out a power-law decay, even for $r \gg \xi^{*}$.

Regarding the critical behavior near the phase transition on the square and triangular lattices, for $k=7$, our results show that the transition is continuous and occurs for $\rho_{2}^{*}=0.917 \pm .015\left(\mu_{c}=5.57 \pm .02\right)$ on the square lattice, and for $\rho_{2}^{*}=0.905 \pm .010\left(\mu_{c}=5.14 \pm 0.05\right)$ on the triangular lattice, where $\mu_{c}$ is the critical chemical potential. For comparison, $\rho_{1}^{*} \approx 0.745$ on the square lattice. On the square lattice, our best estimates of the effective critical exponents differ from the Ising universality class, with exponents $\nu=0.90 \pm 0.05, \beta / \nu=0.22 \pm 0.07$, $\gamma / \nu=1.56 \pm 0.07$ and $\alpha / \nu=0.22 \pm 0.07$. However, it appears that these are only effective exponents, and may be expected to crossover to the Ising universality class at larger length scales. On the triangular lattice, our estimates of critical exponents for the second transition are consistent with those of the three-state Potts model universality class $(\nu=5 / 6, \beta=1 / 9)$.

The plan of the paper is as follows. In Sec. II we define the model precisely, and describe the Monte Carlo algorithm used. In Sec. III we use this algorithm to show that at high activities, the nematic phase is unstable to creation of bubbles of HDD phase, and that the decay of the nematic order parameter to zero is welldescribed quantitatively by the classical nucleation theory of Kolmogorov-Johnson-Mehl-Avrami. In Sec. IV] we study different properties of the HDD phase: the two point correlations, cluster size distributions, susceptibility, size distribution of structures that we call 'stacks', and the formation of bound states of vacancies. The critical behavior near the second transition from the nematic phase to the HDD phase is studied in Sec. $\nabla$ for both the square and triangular lattices, by determining the numerical values of the critical exponents. SectionVI summarizes the main results of the paper, and discuss some possible extensions.

\section{MODEL AND THE MONTE CARLO ALGORITHM}

For simplicity, we first define the model on the square lattice. Generalization to the triangular lattice is straightforward. Consider a square lattice of size $L \times L$ with periodic boundary conditions. A $k$-mer, can be either horizontal (x-mer) or vertical (y-mer). A lattice site can have at most one $k$-mer passing through it. An activity $z=e^{\mu}$ is associated with each $k$-mer, where $\mu$ is the chemical potential.

The Monte-Carlo algorithm we use is defined as follows (this was reported earlier in a conference [21]): given a valid configuration, first, all $\mathrm{x}$-mers are removed without moving any of the y-mers. Each row now consists of sets of contiguous empty sites, separated from each other by sites occupied by y-mers. The lattice is now reoccupied with $\mathrm{x}$-mers. In the grand canonical ensemble, this can be done independently in each row, and the problem reduces to that of occupying an interval of some given length $\ell$ of a one dimensional lattice with k-mers with correct probabilities.

Let the grand canonical partition function of a system of hard rods on a one dimensional lattice of $\ell$ sites with open boundary conditions be denoted by $\Omega_{o}(z ; \ell)$. The probability that the left most site is occupied by the left most site of a $\mathrm{x}$-mer is $p_{\ell}=z \Omega_{o}(z ; \ell-k) / \Omega_{o}(z ; \ell)$. If not occupied, we consider the neighbor to the right and reduce the number of lattice sites by one. If occupied, we move to the $(k+1)^{t h}$ neighbor and reduce the length of the interval by $k$.

The partition functions $\Omega_{o}(z ; \ell)$ obeys the simple recursion relation $\Omega_{o}(z ; \ell)=z \Omega_{o}(z ; \ell-k)+\Omega_{o}(z ; \ell-1)$, for $\ell \geq k$, and $\Omega_{o}(z ; \ell)=1$ for $\ell=0,1, \ldots, k-1$. The solution of this recursion relation is $\Omega_{o}(z ; \ell)=\sum_{i=1}^{k} a_{i} \lambda_{i}^{\ell}$, where $\lambda_{i}$ 's are independent of $\ell$. The $a_{i}$ 's are determined by the boundary conditions $\Omega_{o}(z ; \ell)=1$ for $\ell=0,1, \ldots, k-1$.

With periodic boundary conditions, the recursion relations have to be modified. Let $\Omega_{p}(z ; \ell)$ be the partition function of a one dimensional lattice of length $\ell$ with periodic boundary conditions. It is easy to see that $\Omega_{p}(z ; \ell)=k z \Omega_{o}(z ; \ell-k)+\Omega_{o}(z ; \ell-1)$. We use a list of stored values of the relevant probabilities $\left\{p_{\ell}\right\}$ for all $\ell=1, \ldots, L$, to reduce the computation time.

Following the evaporation of and re-occupation by $\mathrm{x}$-mers, we repeat the procedure with y-mers. Keeping $\mathrm{x}$-mers unmoved, all $\mathrm{y}$-mers are evaporated and the columns are then reoccupied with y-mers. A Monte Carlo move corresponds to one set of evaporation and re-occupation of both $\mathrm{x}$-mers and $\mathrm{y}$-mers. It is straightforward to see that the algorithm is ergodic, and satisfies the detailed balance condition.

The algorithm is easily parallelizable since the evaporation and reoccupation of $\mathrm{x}$-mers in any row (column) is independent of the other rows (columns). For the larger system sizes $(L>400)$, we used a parallelized version of the computer program. This enables us to study the critical behavior at the second transition for system sizes up to $L=952$, properties of the HDD phase away from the transition point for system sizes up to $L=2576$, and probe densities up to $\rho=0.995$. At these high densities, we ensure equilibration by checking that the long time behavior of the system is independent of the initial 
preparation. For this, we used two different initial conditions, one in which all sites are occupied by $\mathrm{x}$-mers and the other in which one half of the lattice contains $\mathrm{x}$-mers and the other half only y-mers.

\section{METASTABILITY OF THE NEMATIC PHASE FOR LARGE ACTIVITIES}

We first verify that, for large activities, the nematic phase is unstable to the growth of the HDD phase. In Fig. 11(a)-(c), we show snapshots of the system of rods of length $k=7$ in equilibrium, on a square lattice at low, intermediate and high densities. For the high-density snapshot, the initial configuration had full nematic order, but the system relaxed to a disordered phase. A similar disordered phase is also seen for the triangular lattice at high densities (see Fig. 2).

In Fig. 3. we show the temporal evolution of the order parameter $Q$, defined by $Q=\left\langle n_{h}-n_{v}\right\rangle /\left\langle n_{h}+n_{v}\right\rangle$, where $n_{h}$ and $n_{v}$ are the number of x-mers and y-mers respectively. For all values of $\mu$, the initial configuration had full nematic order. For $\mu=3.89$, at large times, the system relaxes to an equilibrium state with a finite nematic order. However, for larger $\mu=7.60$, the nematic order decreases with time to zero. Interestingly, we find that the average lifetime of the metastable state decreases with increasing system size, and saturates to a $L$ independent value for $L \gtrsim 200$ (see Fig. 3).

Naively, faster relaxation for larger systems may appear unexpected, but is easily explained using the wellknown nucleation theory of Kolmogorov-Johnson-MehlAvrami [22, 23]. We assume that critical droplets of the stable phase are created with a small uniform rate $\epsilon$ per unit time per unit area, and once formed, the droplet radius grows at a constant rate $v$. Then, the probability that any randomly chosen site is still not invaded by the stable phase is given by $\exp \left[-\epsilon \int_{0}^{t} d t^{\prime} V\left(t^{\prime}\right)\right]$, where $V\left(t^{\prime}\right)$ is the area of the region such that a nucleation event within this area will reach the origin before time $t^{\prime}$. The area $V\left(t^{\prime}\right)$ is given by $V\left(t^{\prime}\right)=\pi v^{2} t^{\prime 2}$ when the droplet is smaller than the size of the lattice. For time $t^{\prime}$ greater than this characteristic time $t^{*}$, we have $V\left(t^{\prime}\right)=L^{2}$. If the droplet does not grow equally fast in all directions, we take suitably defined average over directions to define $v^{2}$. Thus, we obtain

$$
\begin{aligned}
Q(t) & =\exp \left[-\frac{\pi}{3} \epsilon v^{2} t^{3}\right], \text { for } t<t^{*} \\
& =\exp \left[-\pi \epsilon v^{2} t^{* 2}\left(t-\frac{2 t^{*}}{3}\right)\right], \text { for } t>t^{*}
\end{aligned}
$$

We see that with this choice, both $Q(t)$ and its derivative are continuous at $t=t^{*}$. Since $V\left(t^{\prime}\right)$ should tend to $L^{2}$ for large $t^{\prime}$, we get the crossover scale $t^{*}$ given by

$$
t^{*}=\frac{L}{v \sqrt{\pi}} .
$$

The crossover lattice size $L^{*}$ beyond which the average lifetime of the metastable state becomes independent of $L$ can then be estimated from the above to be

$$
L^{*} \sim\left(\frac{3 \sqrt{\pi} v}{\epsilon}\right)^{1 / 3} .
$$

Fitting the numerical data in Fig. 3 to Eq. (1) we obtain $\epsilon=(2.1 \pm 0.2) \times 10^{-10}$ and $v=(5.5 \pm 0.7) \times 10^{-5}$ for $\mu=7.60$. From Eq. (3), we then obtain the crossover scale $L^{*} \sim 110$, of the same order as the numerically observed value of $L^{*} \sim 200$. The difference is presumably due to simplifying approximations made in the theory, e.g., neglecting the dependence of the mean velocity of growth on the direction of growth, or the curvature of the interface, etc.

We can also estimate $v$ directly from simulations of a system with an initial configuration where half the sample is in the nematic phase and the other half is in the equilibrium disordered phase at that $\mu$. For $\mu=7.60$, we find that this velocity increases slowly with $L$, and tends to a limiting value $\approx 1.0 \times 10^{-4}$ for $L \geq 784$, reasonably close to the velocity obtained from fitting data to Eq. (1). For decreasing chemical potential $\mu$, we find that both the velocity $v$ and nucleation rate $\epsilon$ increase.

\section{NATURE OF THE HIGH-DENSITY DISORDERED PHASE}

There is a one-to-one correspondence between fully packed k-mer configurations and a restricted solid on solid height model with vector-valued heights [24, 25]. The height fluctuations at large length scales are welldescribed by a gaussian model, and at full packing the orientation-orientation correlation function decays as a power-law with distance. The exponent of this power law has been estimated for the case $k=3$ by exact diagonalization studies [26]. If these correlations are not destroyed by small density of vacancies for large $k$, then the correlations in the HDD phase would be long-ranged, qualitatively different from the known exponential decay of correlations in the LDD phase. In this section, we test this possibility by studying the susceptibility $\chi$, the order parameter correlation function $C_{S S}(i, j)$, the cluster size distribution $F(s)$, and the size distribution of structures that we call stacks. We also examine the formation of bound states of vacancies.

The susceptibility is defined as $\chi=L^{2}\left\langle\left(n_{h}-\right.\right.$ $\left.\left.n_{v}\right)^{2}\right\rangle /\left\langle n_{h}+n_{v}\right\rangle^{2}$, where $n_{h}$ and $n_{v}$ are the number of $\mathrm{x}$-mers and y-mers. Figure 4 shows the variation of $\chi$ with $L$, for three different values of $\mu$ in the HDD phase. $\chi$ tends to a finite non-zero value for large $L$, hence, if the correlations are a power law, then the decay exponent is larger than 2. From the central limit theorem, it follows that the order parameter $Q$ should scale as $L^{-1}$. This is confirmed in the inset of Fig. 4, where the scaled probability distributions for different $L$ 's collapse onto one curve when plotted against $Q L$. 


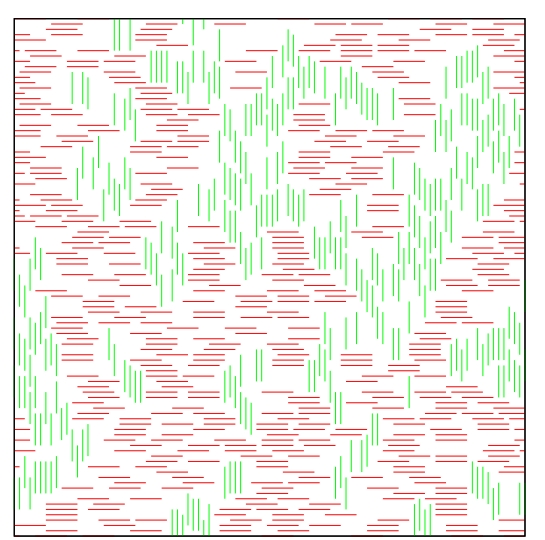

(a)

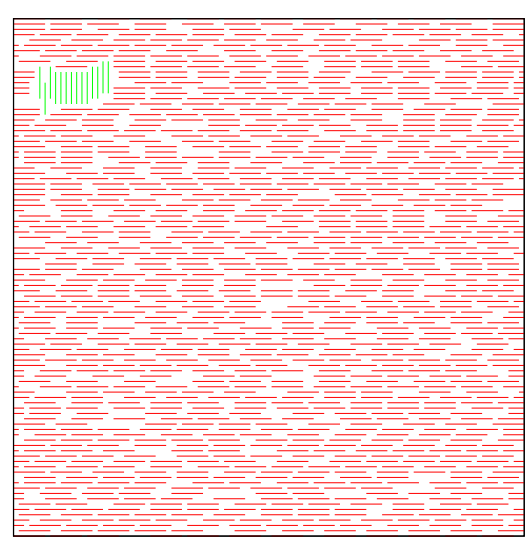

(b)

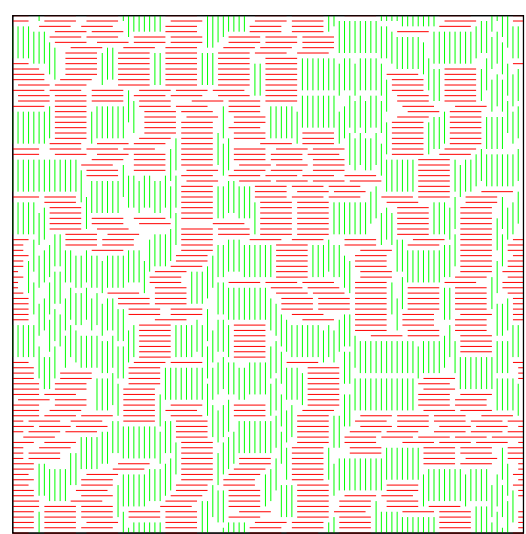

(c)

FIG. 1: (Color online) Typical configurations of the system in equilibrium at densities (a) $\rho \approx 0.66(\mu=0.41)(\mathrm{b}) \rho \approx 0.89$ $(\mu=4.82)$, and (c) $\rho \approx 0.96(\mu=7.60)$ on a square lattice. Here, $k=7$ and $L=98$.

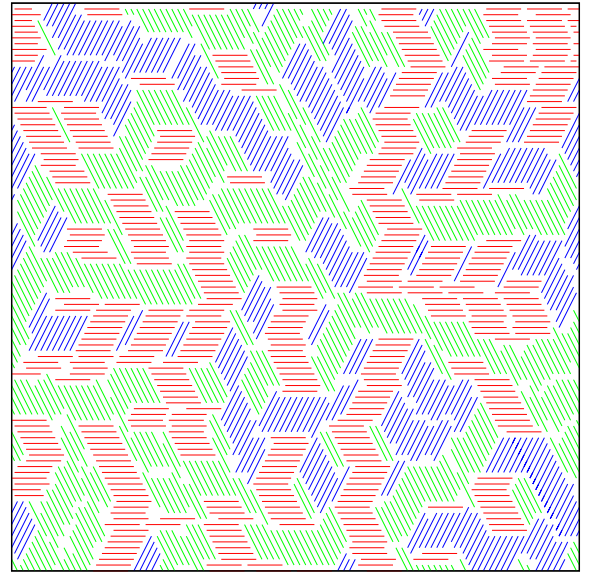

FIG. 2: (Color online) A typical configuration of the system in equilibrium at density $\rho \approx 0.96(\mu=7.60)$ on a triangular lattice. Here, $k=7$ and $L=98$.

The order parameter correlation function $C_{S S}(i, j)$ is defined as follows. Given a configuration, we assign to each site $(i, j)$ a variable $S_{i, j}$, where $S_{i, j}=1$ if $(i, j)$ is occupied by an x-mer, $S_{i, j}=-1$ if $(i, j)$ is occupied by an y-mer, and $S_{i, j}=0$ if $(i, j)$ is empty. Then,

$$
C_{S S}(i, j)=\left\langle S_{0,0} S_{i, j}\right\rangle .
$$

Figure 5 shows the variation of $C_{S S}(r)$ with separation $r$ along the $x$ - and $y$ - axes, for different chemical potentials and systems sizes. In the HDD phase, the correlation function has an oscillatory dependence on distance with period $k$, and for $r \gg k$, appears to decrease as a power law $r^{-\eta}$, with $\eta>2$. Given the limited range of $r$ available $7 \ll r \ll L / 2$, it is difficult to get an accurate estimate of the exponent $\eta$.

The long-range correlations in the HDD phase are better studied by looking at the large-scale properties of con-

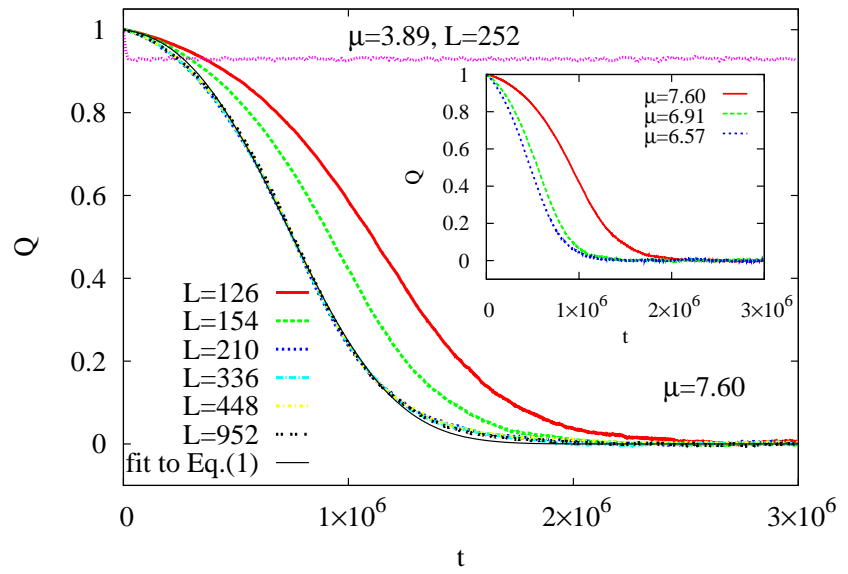

FIG. 3: (Color online) Decay of the order parameter $Q$ for the square lattice as a function of time (Monte Carlo steps), starting from a fully ordered state for two different values of $\mu: \mu=3.89(\rho \approx 0.867)$, and $\mu=7.60(\rho \approx 0.957)$. The best fit of the data to Eq. (1) with additional subleading terms is also shown. Inset: Data for different chemical potentials, all corresponding to HDD phase for $L=154$ and $k=7$. The densities corresponding to these values of $\mu$ are approximately $0.957,0.948,0.941$.

nected clusters of parallel rods. For instance, it is known that the exponent characterizing the decay of cluster size distribution of critical Fortuin-Kasteleyn clusters [27] in the $q$-state Potts model [28, 29] has a non-trivial dependence on $q$. We denote all sites occupied by x-mers by 1 and the rest by zero. For our problem, we define a cluster as a set of 1 's connected by nearest neighbor bonds. Let $F(s)$ be the probability that a randomly chosen 1 belongs to a cluster of $s$ sites. Clearly, $F(s)$ is zero, unless $s$ is a multiple of $k$. Let the cumulative distribution function be $F_{\text {cum }}(s)=\sum_{s^{\prime}=1}^{s} F\left(s^{\prime}\right)$. 


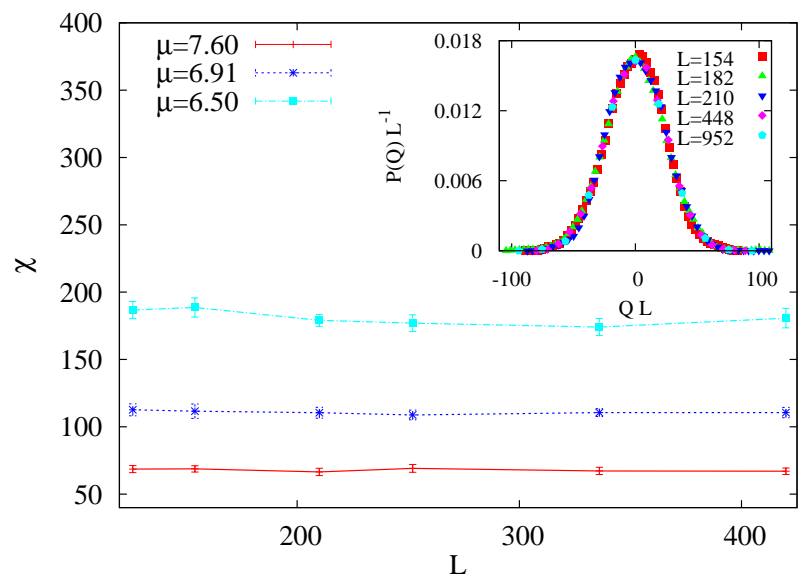

FIG. 4: (Color online) Susceptibility $\chi$ for the square lattice as a function of $L$ for three values of $\mu$, all in the HDD phase. There is no anomalous dependence on $L$. Inset: The scaled probability distribution for the order parameter $P(Q)$ for different $L$ 's collapse when plotted against $Q L$. The data are for $\mu=5.95$.

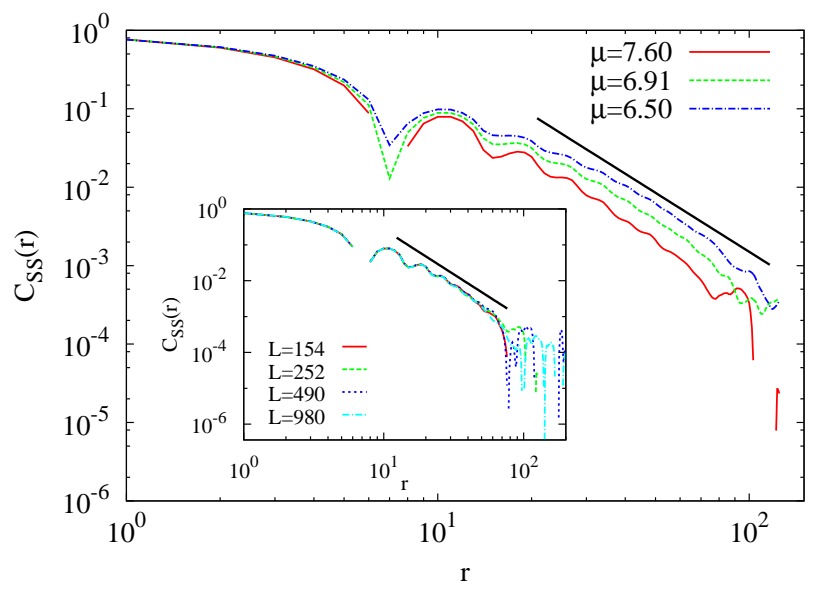

FIG. 5: (Color online) Order parameter correlations $C_{S S}(r)$ for the square lattice as a function of $r$, measured along the $x$ and $y$-axes, for three different values of $\mu$, all of them larger than $\mu_{c} \approx 5.57$. The system size is $L=252$. Inset: The dependence of $C_{S S}(r)$ on $L$ is shown for $\mu=7.60$. The solid lines are power laws $r^{-2.5}$, intended only as guides to the eye.

In Fig. 6, we plot $F_{\text {cum }}(s)$ in the HDD phase for different system sizes on the square lattice. We find that for intermediate range of $s$, for $10^{3} \ll s \ll 10^{6}$, $F_{\text {cum }}(s) \simeq A s^{1-\tau}$, with $\tau<1$. For $\mu=7.60$, we estimate the numerical values to be $A=0.037$ and $\tau=0.762$. For small system sizes (up to $L=1568$ ),$F_{\text {cum }}(s)$ has a system-size dependent cutoff. The $L$-independent cutoff $s^{*}$ is determined by the condition $A s^{* 1-\tau} \approx 1$, giving $s^{*} \approx 1.04 \times 10^{6}$. The density of 1 's being roughly 0.48 , we expect to observe $s^{*}$ only when $L$ exceeds a characteristic length scale $\xi^{*} \sim 1400$. This is indeed seen in Fig. 6.

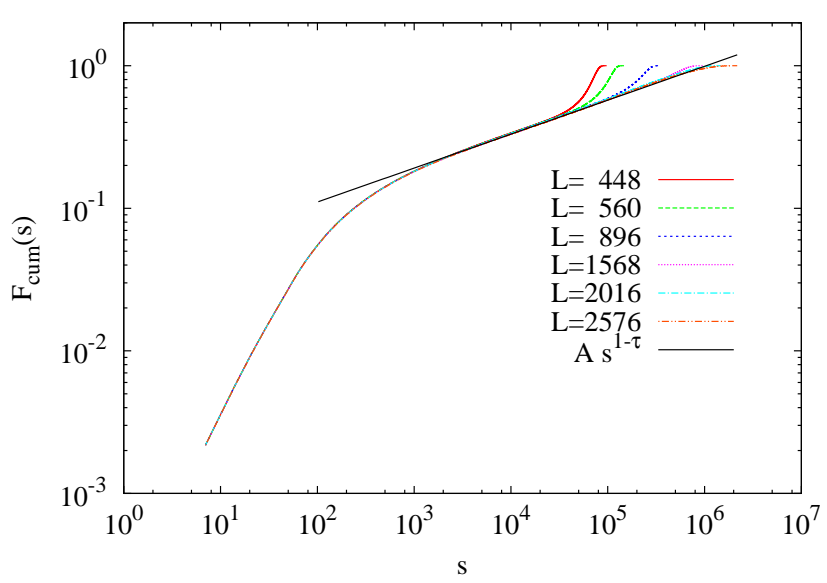

FIG. 6: (Color online) $F_{\text {cum }}(s)$, the probability that a randomly chosen 1 (a site occupied by a x-mer) belongs to a connected cluster of size $\leq s$, in the HDD phase $(\mu=7.60)$ for different system sizes. The data are for the square lattice.

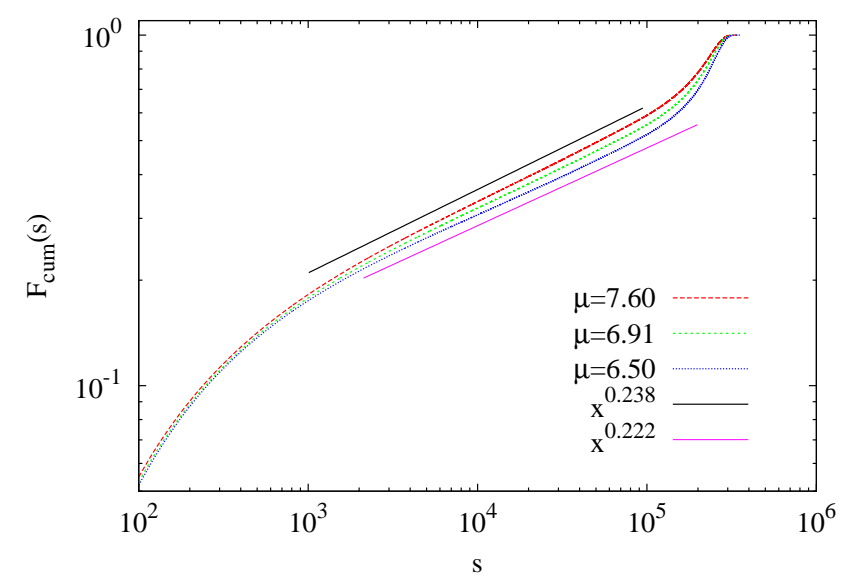

FIG. 7: (Color online) $F_{\text {cum }}(s)$, the probability that a randomly chosen 1 (a site occupied by a x-mer) belongs to a connected cluster of size $\leq s$, for different values of $\mu$, all corresponding to the HDD phase. The curves appear to have weakly density dependent power-law exponents.

In the HDD phase, $F_{\text {cum }}(s)$ depends weakly on $\mu$ (see Fig. 7). The power law exponent $\tau$ is estimated to be $0.778(\mu=6.50), 0.767(\mu=6.91)$ and $0.762(\mu=7.60)$. It appears that $\tau$ decreases slowly with increasing $\mu$, while $s^{*}$ decreases with increasing $\mu$.

One qualitative feature of the HDD phase is the appearance of large groups of parallel rods, worm-like in appearance, nearly aligned in the transverse direction. This is clearly seen in Fig. 1(c). We call these groups stacks. To be precise, we define a stack as follows: two neighboring parallel $k$-mers are said to belong to the same stack if the number of nearest-neighbor bonds between them is greater than $k / 2$. A stack is the maximal cluster of rods that can be so constructed. By this definition, a stack has a linear structure without branching, with some 


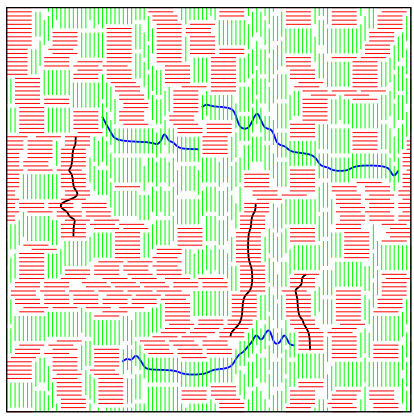

(a)

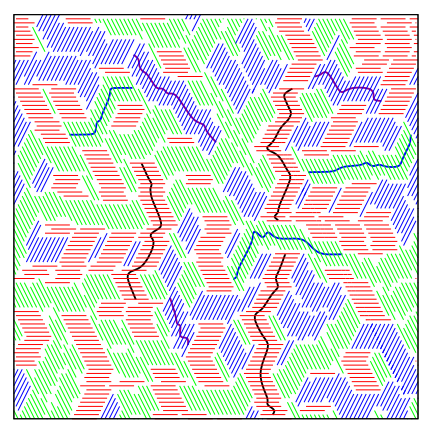

(b)
FIG. 8: (Color online) Some examples of the different types of stacks, shown here as rods joined by wiggly lines, for (a) square lattice and (b) triangular lattice. The snapshots are for $\mu=7.60$, corresponding to the HDD phase. Rods of different orientations are shown in different colors for easy visualization.

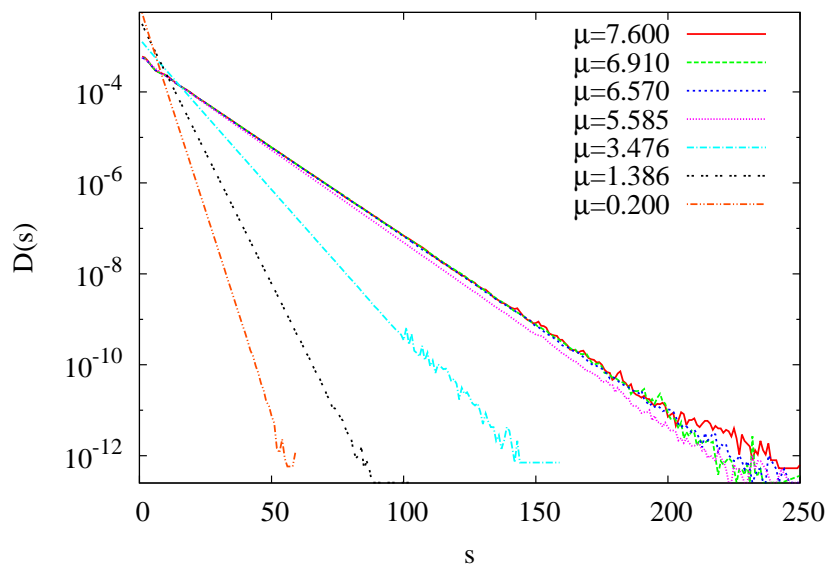

FIG. 9: (Color online) Stack distribution in the LDD phase $(\mu=0.200)$, intermediate density nematic phase $(\mu=3.476)$, HDD phase $(\mu=7.600)$, and at two critical points $(\mu=$ $1.3863,5.57)$ are shown. Data are for $L=280, k=7$, and the square lattice.

transverse fluctuations allowed. Examples of stacks on square and triangular lattices are shown in Fig. 8 . Any given configuration of rods is uniquely broken up into a collection of disjoint stacks.

There are a noticeable number of large stacks in the HDD phase. We measured the stack size distribution $D(s)$, the number of stacks of size $s$ per site of the lattice, in all the three phases and at the transition points (see Fig. 9). Interestingly, we found that this distribution is nearly exponential in all the three phases, as well as at the critical points, and there is no indication of any power-law tail in this function. In the HDD phase, the mean stack size is approximately 12 , for both square and triangular lattices, and is only weakly dependent on the density.

It was suggested in Ref. [12] that the second phase

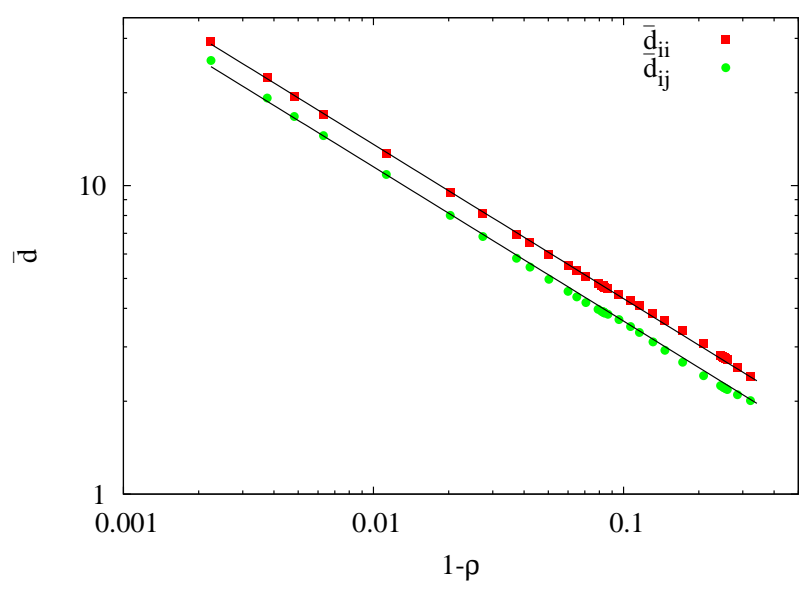

FIG. 10: (Color online) The average spacing between vacancies $\bar{d}_{i i}$ and $\bar{d}_{i j}$, on the square lattice as a function of density $\rho$. The solid lines show the functions $K(1-\rho)^{-1 / 2}$, for $K=1.36$ and 1.15. The data are for $L=168$ and $k=7$.

transition may be viewed as a binding-unbinding transition of $k$ species of vacancies. For studying such a characterization, we break the square lattice into $k$ sublattices. A site $(x, y)$ belongs to the $i$-th sublattice if $x+y=i(\bmod k)$, where $i=0,1, \ldots, k-1$. In a typical configuration with a low density of vacant sites, it was argued that the vacancies would form bound states of $k$ vacancies, one from each sublattice. The HDD phase can then be described as a weakly interacting gas of such bound states if the typical distance between two bound states is much larger than the mean size of a bound state.

Let $d_{i j}$ be the Euclidean distance between a randomly picked vacant site on the $i$-th sublattice, and the vacant site nearest to it on the $j$-th sublattice. The average of $d_{i j}$, averaged over all pairs $(i j)$, with $i \neq j$, will be denoted by $\bar{d}_{i j}$, and $\bar{d}_{i i}$ will denote the value of $d_{i i}$, averaged over $i$.

In Fig. 10, we show the variation of $\bar{d}_{i j}$ and $\bar{d}_{i i}$ with density $\rho$. We see that $\bar{d}_{i i}$ and $\bar{d}_{i j}$, both vary approximately as $(1-\rho)^{-1 / 2}$, with $\bar{d}_{i i} \approx 1.18 \bar{d}_{i j}$. The data are for $L=168$ and $k=7$. There is no noticeable dependence of the data on $L$. We see no signature of $\bar{d}_{i j}$ saturating to a finite value, for the densities up to 0.995 , when $\bar{d}_{i j} \simeq 35$.

We conclude that the bound state, if exists at all, is very weakly bound. Near $\rho_{2}^{*}$, the typical spacing between vacancies is much less than the size of the bound state, and the transition can not be treated as bindingunbinding transition when the average distance between bound states becomes comparable to their size.

\section{CRITICAL BEHAVIOR NEAR THE SECOND TRANSITION}

We now discuss the critical behavior near the second transition. Several thermodynamic quantities are of interest. We define the nematic order parameter $m$ 
as follows. For the square lattice, $m=\left(n_{h}-n_{v}\right) / N$, where $n_{h}$ and $n_{v}$ are the number of lattice sites occupied by x-mers and y-mers respectively, and $N$ is the total number of lattice sites. For the triangular lattice, $m=\left(n_{1}+\omega n_{2}+\omega^{2} n_{3}\right) / N$, where $\omega$ is the complex cuberoot of unity, and $n_{1}, n_{2}, n_{3}$ are the number of sites occupied by $k$-mers oriented along the three allowed directions. The density $\rho$ is defined by the fraction of sites that are occupied by the $k$-mers. The order parameter $Q$, its second moment $\chi$, compressibility $\kappa$, and Binder cumulant $U$ are defined as

$$
\begin{aligned}
Q & =\frac{\langle|m|\rangle}{\langle\rho\rangle}, \\
\chi & =\frac{L^{2}\left\langle|m|^{2}\right\rangle}{\langle\rho\rangle^{2}}, \\
\kappa & =L^{2}\left[\left\langle\rho^{2}\right\rangle-\langle\rho\rangle^{2}\right], \\
U & =1-\frac{\left\langle|m|^{4}\right\rangle}{a\left\langle|m|^{2}\right\rangle^{2}},
\end{aligned}
$$

where $a=3$ for square lattice and $a=2$ for triangular lattice. $Q$ is zero in the LDD and HDD phases and nonzero in the nematic phase.

The data used for estimating the critical exponents are for $k=7$, and for five different system sizes $L=$ $154,210,336,448$, and 952 for the square lattice and $L=210,336,448$, and 560 for the triangular lattice. The system is equilibrated for $10^{7}$ Monte Carlo steps for each $\mu$, following which the data are averaged over $3 \times$ $10^{8}$ Monte Carlo steps. These times are larger than the largest autocorrelation times that we encounter obtained by measuring the autocorrelation function

$$
A_{Q Q}(t)=\frac{\langle Q(\tau) Q(\tau+t)\rangle}{\left\langle Q^{2}\right\rangle},
$$

where the averaging is done over the reference time $\tau$. The function $A_{S S}(t)$ is defined similarly. The largest autocorrelation time is for the largest density and is close to $2.2 \times 10^{5}$ Monte Carlo steps (see Fig. 11). To estimate errors, the measurement is broken up into 10 statistically independent blocks.

The quantities in Eq. (5) are determined as a function of $\mu$ using Monte Carlo simulations. The nature of the second transition from the ordered nematic phase to the HDD phase is determined by the singular behavior of $U$, $Q, \chi$, and $\kappa$ near the critical point. Let $\epsilon=\left(\mu-\mu_{c}\right) / \mu_{c}$, where $\mu_{c}$ is the critical chemical potential. The singular behavior is characterized by the critical exponents $\nu, \beta$, $\gamma$, and $\alpha$, defined by $Q \sim(-\epsilon)^{\beta}, \epsilon<0, \chi \sim|\epsilon|^{-\gamma}$ and $\kappa \sim|\epsilon|^{-\alpha}$, and $\xi^{*} \sim|\epsilon|^{-\nu}$, where $\xi^{*}$ is the correlation length and $|\epsilon| \rightarrow 0$. The exponents are obtained by finite size scaling of the different quantities near the critical point:

$$
\begin{aligned}
& U \simeq f_{U}\left(\epsilon L^{1 / \nu}\right) \\
& Q \simeq L^{-\beta / \nu} f_{Q}\left(\epsilon L^{1 / \nu}\right) \\
& \chi \simeq L^{\gamma / \nu} f_{\chi}\left(\epsilon L^{1 / \nu}\right) \\
& \kappa \simeq L^{\alpha / \nu} f_{\kappa}\left(\epsilon L^{1 / \nu}\right)
\end{aligned}
$$

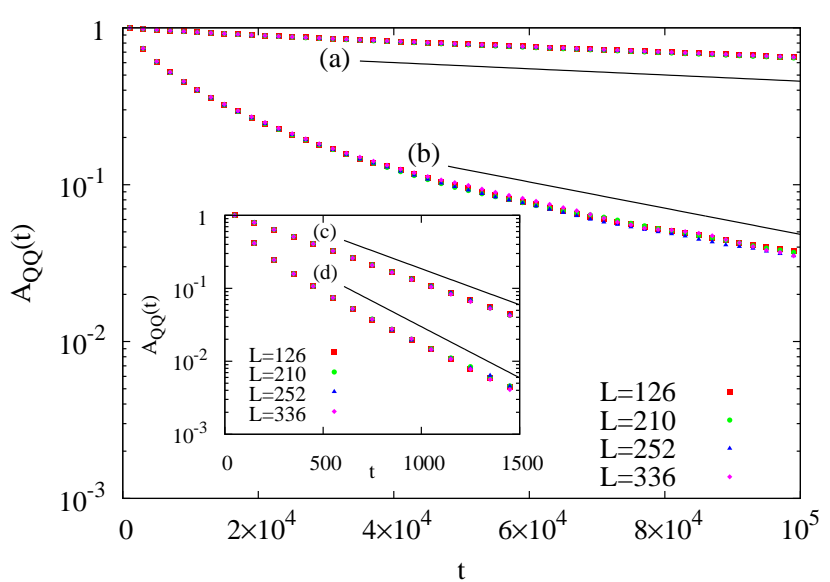

FIG. 11: (Color online) The temporal variation of the autocorrelation functions for (a) the global order parameter $Q$ and (b) the local order parameter $S$. The data are for $\mu=7.60$ and the autocorrelation times corresponding to the solid lines are (a)220000 and (b) 52000. Inset: Data as above but in the LDD phase $(\mu=0.20)$, with (c) corresponding to $A_{Q Q}(t)(\mathrm{d})$ corresponding to $A_{S S}(t)$. The autocorrelation times are (c) 440 and (d) 310. All data are for $k=7$.

where $f_{U}, f_{Q}, f_{\chi}$, and $f_{\kappa}$ are scaling functions.

\section{A. Square lattice}

We first present results for the square lattice. The data for the Binder cumulant $U$ for different system sizes intersect at $\mu_{c}=5.57 \pm .02$ (see Fig. 12). The density at this value of chemical potential is $\rho_{2}^{*}=0.917 \pm .015$, consistent with the variational estimate $0.87 \leq \rho_{2}^{*} \leq 0.93$ in Ref. [17]. The value of $U$ at the transition lies in the range 0.56 to 0.59 . This is not very different from the value for the Ising transition $U_{c} \approx 0.61[30]$. The data for different system sizes collapse when scaled as in Eq. (7a) with $\nu=0.90 \pm .05$ (see inset of Fig. 12). To compare with the first transition from the LDD phase to the nematic phase, $\rho_{1}^{*}=0.745 \pm 0.010$, and the numerical estimate for the exponent $\nu$ is consistent with the known exact Ising value 1 [14].

The data for order parameter, $\chi$ and $\kappa$ for different system sizes are shown in Figs. 13] [14 and 15] respectively. $Q$ decreases to zero at high densities. Our best estimates of effective critical exponents are $\beta / \nu=0.22 \pm 0.07$ (see inset of Fig. 13). $\gamma / \nu=1.56 \pm 0.07$ (see inset of Fig. 14), and $\alpha / \nu=0.22 \pm 0.07$ (see inset of Fig. 15). The estimated error bars are our subjective estimates, based on the goodness of fit. These differ substantially from the values of the exponents of the two dimensional Ising model $(\nu=1, \beta=1 / 8, \gamma=7 / 4, \alpha=0)$. However, as discussed in Sec. IV, it seems like there is a characteristic length scale $\xi^{*} \sim 1400$ in the HDD phase, and we cannot say much about the asymptotic value the critical exponents at length scales $L \gg \xi^{*}$. 


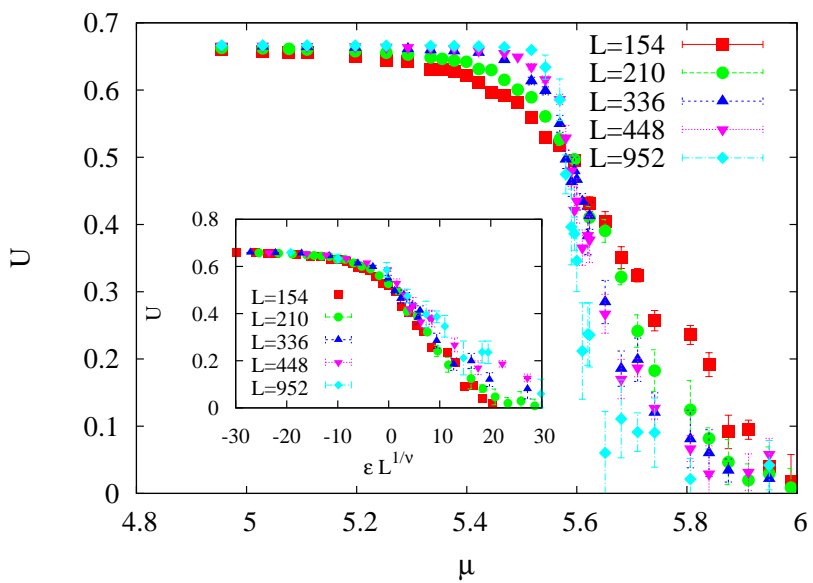

FIG. 12: (Color online) The Binder cumulant $U$ as a function of chemical potential $\mu$ for different lattice sizes of a square lattice. The curves intersect at $\mu_{c}=5.57 \pm .02$. Inset: Data collapse for square lattices when $U$ is plotted against $\epsilon L^{1 / \nu}$ with $\nu=0.90$ and $\epsilon=\left(\mu-\mu_{c}\right) / \mu_{c}$.

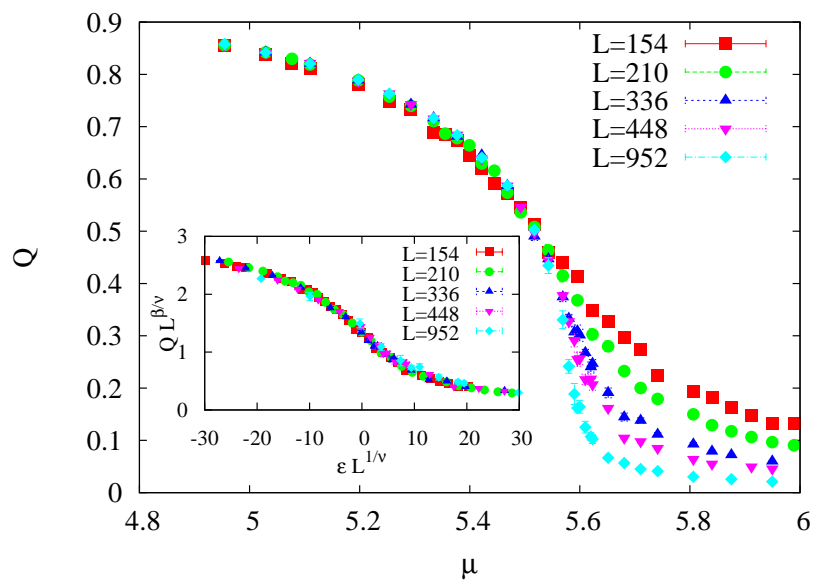

FIG. 13: (Color online) The variation of the order parameter $Q$ with chemical potential $\mu$ for different systems sizes of a square lattice. Inset: Data collapse for square lattices when scaled $Q$ is plotted against $\epsilon L^{1 / \nu}$ with $\nu=0.90, \beta / \nu=0.22$ and $\epsilon=\left(\mu-\mu_{c}\right) / \mu_{c}$.

\section{B. Triangular lattice}

For the triangular lattice, we find that the second transition is continuous with $\mu_{c}=5.147 \pm .050$ and $\rho_{2}^{*}=.905 \pm .010$. The data for $U, Q, \chi$, and $\kappa$ for different system sizes collapse onto one scaling curve when scaled as in Eq. (7) with exponents that are indistinguishable from those of the three state Potts model (see Fig. 16) $(\nu=5 / 6, \beta=1 / 9, \gamma=13 / 9$ and $\alpha=1 / 3)$.

As in the case of the square lattice, we probe the correlations in the triangular lattice by looking at the largescale properties of connected clusters of parallel rods. We denote all sites occupied by horizontal rods by 1 and the

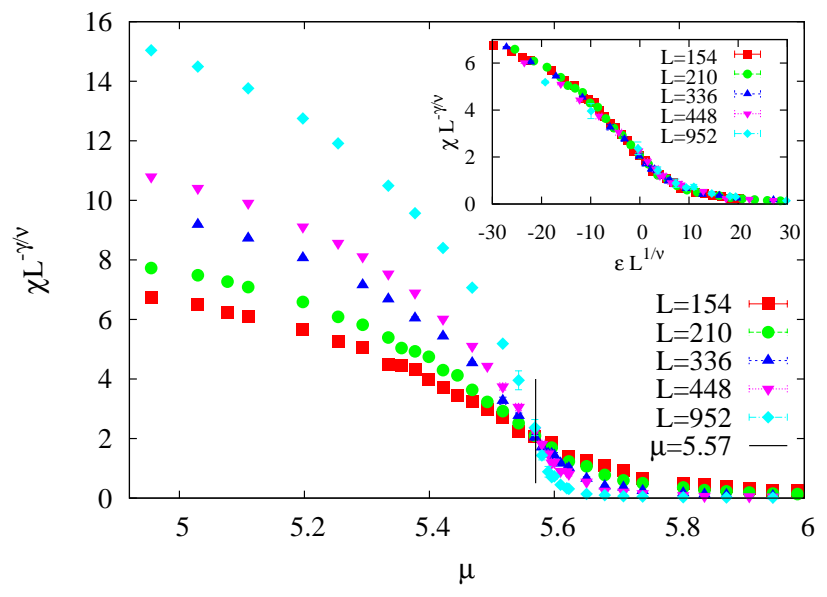

FIG. 14: (Color online) The variation of $\chi$, the mean of the square of the order parameter, with chemical potential $\mu$ for different system sizes of a square lattice. The curves cross at $\mu_{c}$ when $\chi$ is scaled by $L^{-\gamma / \nu}$, with $\gamma / \nu=1.56$. Inset: Data collapse for square lattices when $\chi L^{-\gamma / \nu}$ is plotted against $\epsilon L^{1 / \nu}$ with $\nu=0.90$, and $\epsilon=\left(\mu-\mu_{c}\right) / \mu_{c}$.

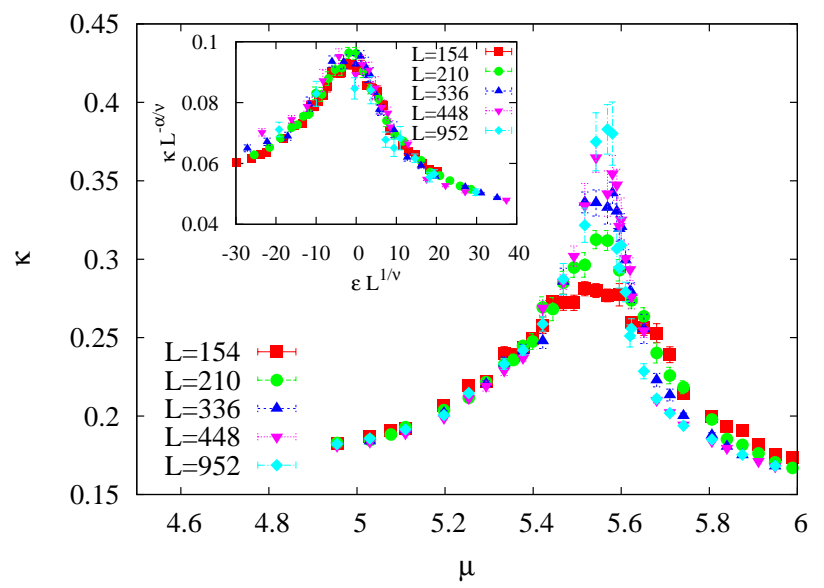

FIG. 15: (Color online) The variation of compressibility $\kappa$ with chemical potential $\mu$ for different system sizes of a square lattice. Inset: Data collapse for square lattices when the scaled $\kappa$ is plotted against $\epsilon L^{1 / \nu}$ with $\nu=0.90, \alpha / \nu=0.22$, and $\epsilon=\left(\mu-\mu_{c}\right) / \mu_{c}$.

rest by zero. In Fig. $17 F(s)$, the probability that a randomly chosen 1 belongs to a cluster of $s$ sites, is shown for different system sizes $L$ in the HDD phase. Unlike the square lattice case, here there is no extended regime of $s$ where $F(s)$ seems to grow as a power of $s$. This suggests that for the triangular lattice, the HDD and LDD phases are qualitatively similar, and the HDD phase has a finite correlation length $\sim 60$. 

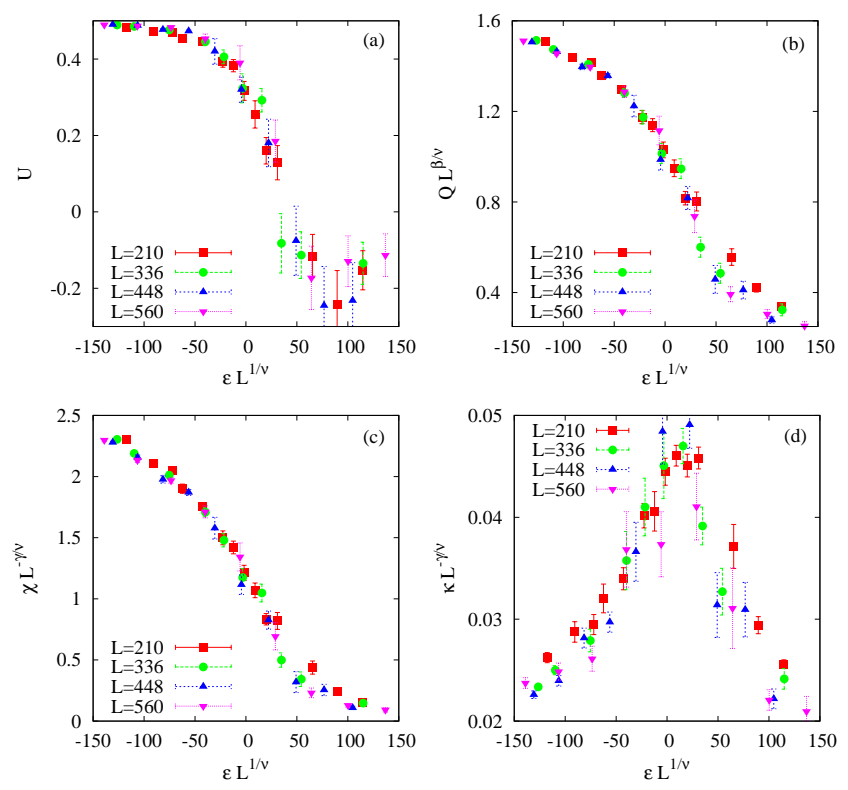

FIG. 16: (Color online) Finite size scaling of the triangular lattice data of (a) U, (b) Q, (c) $\chi$, and (d) $\kappa$ as in Eq. (7) with $\nu=5 / 6, \beta / \nu=2 / 15, \gamma / \nu=26 / 15$ and $\alpha / \nu=2 / 5$. The critical chemical potential is $\mu_{c}=5.147$.

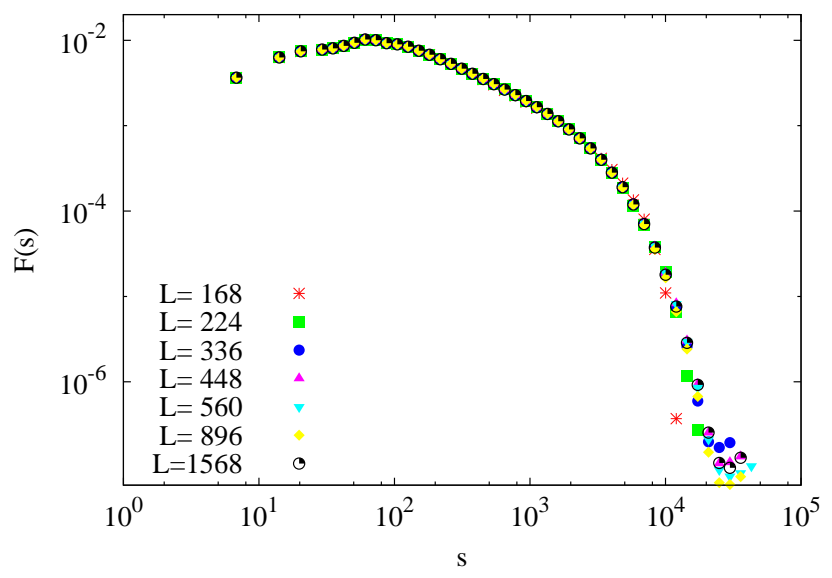

FIG. 17: (Color online) $F(s)$, the probability that a randomly chosen site belongs to a $s$-site connected cluster of horizontal rods, in the HDD phase for different system sizes. The data are for the triangular lattice for $\mu=7.60$.

\section{SUMMARY AND DISCUSSION}

In this paper, we studied the problem of hard, rigid rods on two dimensional square and triangular lattices, using an efficient algorithm that is able to overcome jamming at high densities. The algorithm is more efficient than algorithms with only local moves. In addition to overcoming jamming at high packing densities, it is easily parallelized, which makes it suitable for studying hard-core systems with other particle shapes, and also in higher dimensions.
We showed the existence of a second transition from the ordered nematic phase to a disordered phase as the packing density is increased. By studying the order parameter, its second moment, compressibility and Binder cumulant, we find that the second transition is continuous on both square and triangular lattices. We also investigated the nature of correlations in the HDD phase by measuring distribution of connected clusters of parallel rods, as well as the distribution of stacks.

We are not able to give a very clear answer to the question whether the HDD and LDD phases are qualitatively different, or not. But the available evidence suggests that while the HDD phase has a large correlation length $\xi^{*}$, it is not qualitatively different. This is based on the evidence that vacancies in the HDD phase do not form a bound state. In that sense, a k-mer system with $k=7$, at high densities is similar to the $k=2$ case, where also, the monomers do not form a bound state, and unbound monomers lead to an exponential decay of correlations at any non-zero vacancy density.

Additional support for this scenario comes from the fact that if the hard-core constraint is relaxed, and two $\mathrm{k}$-mers are allowed to share a site, but with a cost in energy, then exact calculation on an artificial lattice (the random locally tree-like-layered lattice) shows 31] two phase transitions at densities $\rho_{c 1}$ and $\rho_{c 2}$ for a range of values of the repulsive energy. The difference between these critical densities decreases as the repulsive energy is decreased, and below a particular value of the repulsive energy, the intermediate nematic phase disappears. Thus, in this special case, which is the only known exactly solved model of $k$-mers showing two phase transitions, the LDD and HDD phases are the same.

An interesting feature of the HDD phase is the appearance of a large characteristic length scale $\xi^{*} \sim 1400$ on the square lattice, as inferred from the fact that the cluster size distribution seems to follow a power law distribution $F(s) \sim s^{-\tau}$, with $\tau<1$ for $s<\xi^{* 2}$. The amplitude of this power-law term is rather small. This is related to the fact that for the $k$-mer problem, various perturbation series involve terms like $k^{-k}$ [12], which then leads to large correlation lengths. The HDD phase has power-law correlations at least for lengths up to $\xi^{*}$.

For the triangular lattice, the correlation length $\xi^{*}$ is much smaller, as near the critical point, clusters of each type of rods cover only about a third of the sites, which is substantially below the corresponding percolation threshold.

On the square lattice, our best estimates of the numerical values of the critical exponents are different from those of the Ising universality class. However, because the correlation lengths in the HDD phase are large, we cannot rule out a crossover to the Ising universality class at larger length scales. For the triangular lattice with $k=7$, the estimated exponents for second transition are consistent with those of two dimensional 3-state Potts universality class.

We expect that the nature of the transition will not 
depend on the rod length $k$. For example, the first transition at $\rho_{1}^{*}$ is Ising-like, whenever it occurs. Several interesting questions remain. For example, the entropy per site in the fully packed $k$-mer problem is expected to vary as $(c \log k) / k^{2}$, for $k$ large, where $c$ is a constant. The argument in Ref. 12] relates this constant to the coefficient $a$ that appears to the way $\rho_{2}^{*}$ varies with $k$, for large $k$ : $\rho_{2}^{*}=1-a / k^{2}+\ldots$ for large $k$. It would be interesting to determine $c$ and $a$ exactly, or to test these by simulations with larger $k$. Also, further studies are needed to determine if the correlations in the HDD phase decay exponentially for distances greater than $\xi^{*}$. This seems like a promising area for further study.

\section{Acknowledgments}

We would like to thank K. Damle, P. Grassberger, S. Digal, D. Das, P. Ray and G. I. Menon for helpful discussions. The simulations for the larger lattice sizes were carried out on the supercomputing machine Annapurna (Intel Nehalem $2.93 \mathrm{GHz}$ ) at The Institute of Mathematical Sciences.
[1] L. Onsager, Ann. N.Y. Acad. Sci. 51, 627 (1949).

[2] P. J. Flory, Proc. R. Soc. 234, 60 (1956).

[3] R. Zwanzig, J. Chem. Phys. 39, 1714 (1963).

[4] G. J. Vroege and H. N. W. Lekkerkerker, Rep. Prog. Phys. 55, 1241 (1992).

[5] J. P. Straley, Phys. Rev. A 4, 675 (1971).

[6] D. Frenkel and R. Eppenga, Phys. Rev. A 31, 1776 (1985).

[7] M. D. Khandkar and M. Barma, Phys. Rev. E 72, 051717 (2005).

[8] R. L. C. Vink, Euro. Phys. J. B 72, 225 (2009).

[9] O. J. Heilmann and E. Lieb, Commun. Math. Phys. 25, 190 (1972).

[10] R. Dickman, J. Chem. Phys. 136, 174105 (2012).

[11] P. G. de Gennes and J. Prost, The Physics of Liquid Crystals (Oxford University Press, Oxford, 1995).

[12] A. Ghosh and D. Dhar, Euro. Phys. Lett. 78, 20003 (2007).

[13] M. Disertori and A. Giuliani, arXiv:1112.5564 (2012).

[14] D. A. Matoz-Fernandez, D. H. Linares, and A. J. Ramirez-Pastor, Euro. Phys. Lett 82, 50007 (2008).

[15] D. A. Matoz-Fernandez, D. H. Linares, and A. J. Ramirez-Pastor, Physica A 387, 6513 (2008).

[16] D. A. Matoz-Fernandez, D. H. Linares, and A. J. Ramirez-Pastor, J. Chem. Phys. 128, 214902 (2008).

[17] D. H. Linares, F. Romá, and A. J. Ramirez-Pastor, J. Stat. Mech. p. P03013 (2008).

[18] T. Fischer and R. L. C. Vink, Euro. Phys. Lett. 85, 56003
(2009).

[19] B. C. Barnes, D. W. Siderius, and L. D. Gelb, Langmuir 25, 6702 (2009).

[20] D. Dhar, R. Rajesh, and J. F. Stilck, Phys. Rev. E 84, 011140 (2011).

[21] J. Kundu, R. Rajesh, D. Dhar, and J. F. Stilck, AIP Conf. Proc. 1447, 113 (2012).

[22] R. A. Ramos, P. A. Rikvold, and M. A. Novotny, Phys. Rev. B 59, 9053 (1999).

[23] P. A. Rikvold and B. M. Gorman, Annual Reviews of Computational Physics 1, 149 (1994).

[24] C. Kenyon and R. Kenyon, in Proc. of 33rd Fundamentals of Computer Science (FOCS) (IEEE Computer Society, Los Alamitos, CA, USA, 1992), pp. 610-619.

[25] W. P. Thurston, Amer. Math. Monthly 97, 757 (1990).

[26] A. Ghosh, D. Dhar, and J. L. Jacobsen, Phys. Rev. E 75, 011115 (2007).

[27] C. M. Fortuin and P. W. Kasteleyn, Physica 57, 536 (1972).

[28] W. Janke and A. M. J. Schakel, Nuclear Physics B 700[FS], 385 (2004).

[29] Y. Deng, H. W. J. Blöte, and B. Nienhuis, Phys. Rev. B 69, 026123 (2004).

[30] G. Kamieniarz and H. W. J. Blöte, J. Phys. A 26, 201 (1993).

[31] J. Kundu, R. Rajesh, D. Dhar, and J. F. Stilck, in preparation. 